\title{
TECHNIQUES FOR REDUCING SPEEDING BEYOND LICENSURE: YOUNG DRIVERS' PREFERENCES
}

\author{
Yi-Ching Lee, Aditya Belwadi, Dana Bonfiglio, Leif Malm, \& Molly Tiedeken \\ Children's Hospital of Philadelphia, Philadelphia, PA, USA \\ LeeY1@email.chop.edu
}

\begin{abstract}
Summary: Young drivers need continued training and educational efforts beyond licensure. The latest in-vehicle monitoring technologies provide a promising way to monitor and advise driving behaviors in real-time. Literature to-date suggests limited success for changing driving behaviors via the use of in-vehicle monitoring technologies, and teens and parents have mixed perceptions about such devices. We argue that certain reinforcement techniques and parameters may lead to more sustainable behavioral changes. This paper describes the findings of an interview with young drivers on their perspectives of in-vehicle monitoring technologies and a feasibility driving simulator experiment that incorporated key reinforcement techniques. Eighteen young drivers participated in individual semi-structured interviews and 17 participated in the simulator experiment. Participants saw values in having a smartphone application-based system that can monitor their driving and provide positive recognition for safe behaviors and negative alerts for unsafe behaviors. Preliminary behavioral data from the simulator experiment showed mixed results. The findings show promise for incorporating reinforcement techniques in continued education beyond licensure but further research is needed to understand the timing for using such techniques.
\end{abstract}

\section{INTRODUCTION}

Motor vehicle crashes are the leading cause of death for 15- to 20-year-olds (NHTSA, 2014). According to the 2012 data, the relative proportion of speeding-related crashes to all crashes decreases with increasing driver age, with young males (ages 15 to 24) representing the highest involvement (37\%) (NHTSA 2014). Prior research indicates that the most common reasons for speeding are perceived advantages of speeding being larger than the perceived disadvantages as well as psychosocial and personality factors such as sensation seeking (Cestac, Paran, \& Delhomme, 2011; Horvath, Lewis, \& Watson, 2012).

Novice driver crashes decline sharply during the first 6 to 24 months of driving, regardless of the age at which driving begins (Foss, Martell, Goodwin, \& O'Brien, 2011). This pattern indicates and confirms that learning and accumulation of experience during the initial years of driving play a critical role in the decline of crashes, suggesting the need for continued training and educational efforts beyond licensure that can enhance experience and safe driving behaviors among novice drivers.

It is now evident that in-vehicle monitoring and recording systems can provide an objective way to enhance young driver safety and provide continued education (Toledo, Lotan, Taubman-BenAri, \& Grimberg, 2012). Real-time alerts and feedback as well as post-driving summary reports can be generated and used as discussion points with driver instructors or parents. Also, seeing 
records of their own driving patterns provides opportunities for young drivers to reflect and think about their own behaviors and consequences (Simons-Morton et al., 2013). However, studies have reported resistance from young drivers about using such in-vehicle technologies, with them claiming that these technologies are virtual parents and are forms of privacy invasion (GesserEdelsburg \& Guttman, 2013). In addition, although some results are positive, such as increased seatbelt use (Farmer, Kirley, \& McCartt, 2010), more complicated behaviors (e.g., speeding) are difficult to change (McCartt, Farmer, \& Jenness, 2010) and the success of the intervention demanded constant support and monitoring from motivated parents (McGehee, Raby, Carney, Lee, \& Reyes, 2007; Simons-Morton, et al., 2013).

According to the branch of learning theory known as contingency management (Bandura, 1969; Skinner, 1953), performance deficits suggest that the person is aware of the ideal behavior and has the skill to do it but chooses not to engage in such behavior because A) there are too many aversive consequences for doing so (e.g., will be late), B) there are limited positive outcomes for doing so (e.g., no perceived benefit for driving the speed limit), and C) the person is getting positive reinforcement for doing a competing behavior (e.g., acceptance by peers that driving faster is cool). We argue that more refined feedback mechanisms, schedules and mode of delivery of feedback from in-vehicle monitoring systems are needed in order to promote longterm maintenance of positive behaviors. Here we investigated young drivers' preferences for receiving real-time feedback and contents of feedback on speeding behaviors via interviews and a feasibility driving simulator experiment. The current study was designed to better understand the effect of reinforcement techniques on the speeding tendencies of young novice drivers. The findings will be used as the foundation for designing speeding intervention programs that can lead to effective, long-term positive behavioral changes.

\section{METHOD}

\section{Study 1 - Interviews}

We reviewed existing, commercially available smartphone-based applications and device-based systems that can track and monitor driving behaviors. Their features, feedback and reward mechanisms (if available) were organized and used to develop an interview guide (see Table 1).

Participants. Eighteen participants (10 female) were recruited from local high schools and universities. All participants were between the ages of 17-20, with an average age of 19.22 years, and held an unrestricted driver's license for 2.17 years on average.

Table 1. Sections and questions in the interview guide

\begin{tabular}{ll}
\hline Sections & Type of questions \\
\hline \hline Driving Experience & driving routine, type of driving, and behaviors while driving \\
\hline Positive Content & positive recognition/reinforcement (e.g., earning points) when safe behaviors are shown \\
\hline Demographics/Licensing & demographics, licensing status \\
\hline Negative Content & negative alerts/warnings (e.g., losing points) when unsafe behaviors are shown \\
\hline Feedback & mode, frequency, content of feedback \\
\hline
\end{tabular}

Procedures and Data Processing. The interview guide was used to conduct the interviews, and participants were asked to provide examples and elaborations when appropriate. If participants 
had not seen a commercially available in-vehicle monitoring system before, a video or photograph of an exemplar was provided. All interviews, conducted individually, were audiorecorded and conducted in a private conference room. Interviews were transcribed and descriptive summary statistics were used to identify recurrent themes and preferences across study participants as well as to summarize participants' demographic characteristics.

\section{RESULTS - INTERVIEWS}

Responses to the semi-structured interview were analyzed to better understand young drivers' needs and preferences for reinforcement techniques while driving. The majority of participants (94.4\%) responded that they use electronic devices while driving for navigation, music, and for phone calls and texts; $88 \%$ of all participants found these devices distracting.

When participants were asked about the in-vehicle monitoring systems that can help detect dangerous situations and help drivers to drive better, none of them had ever used an in-vehicle monitoring system or application, but two participants $(11.8 \%)$ had heard of them before. After viewing a demonstration of an in-vehicle monitoring system, participants responded to questions regarding their potential use of such systems. Although four participants would not like a system to recognize safe driving, about half $(53.8 \%)$ reported that auditory feedback would be preferential, stating that it would be safer because "you don't have to take your eyes off the road to look at a screen." Furthermore, $23.1 \%$ of participants would like a combination of auditory and haptic feedback to alert safe driving behaviors. For receiving negative feedback about unsafe driving behaviors, $67.6 \%$ preferred auditory feedback, such as a beep, while $17.6 \%$ would prefer a haptic vibration, similar to that of a rumble strip, to alert unsafe driving.

Real-time feedback was preferential for both positive $(75 \%)$ and negative $(82.3 \%)$ feedback. In regards to safe (positive) and unsafe (negative) behaviors to monitor, the top three behaviors to monitor were the same for both contents: following speed limit (positive $-48.1 \%$, negative $32.4 \%$ ), appropriate braking (positive $-18.6 \%$, negative $-22.5 \%$ ), and safe headway distance (positive $-9.8 \%$, negative $-12.7 \%$ ).

Based on the features in existing, commercially available in-vehicle monitoring systems, we asked our participants about the idea of earning (based on safe behaviors) or losing (based on unsafe behaviors) points. The majority of participants $(76.5 \%)$ stated that the points would encourage them to drive better, especially if there is a reward attached to a certain amount of points. Furthermore, $64.7 \%$ of participants stated that negative points should be made up by safe behaviors in order to earn the rewards. The majority of participants stated that they would like to find out their point balance on a smartphone-based application (54.5\%), followed by an email $(21.1 \%)$, a text message $(14.4 \%)$, or on a website $(10 \%)$.

\section{Study 2 - Simulator Study}

Upon completion of the interviews, we designed a feasibility driving simulator experiment that included a few key findings from the interviews: a) drivers would receive feedback messages about their speeding behaviors, b) the feedback was delivered in the form of auditory messages, and c) the feedback messages were presented in real-time. 
Participants. Twenty-two participants (3 pilots, 13 females) were recruited from local high schools and universities as well as subject pools. All participants were between the ages of 1720, held unrestricted driver's licenses, had normal or corrected-to-normal vision, had normal hearing abilities, and spoke English fluently. Seven of them participated in the interviews as well. Two participants were unable to complete the simulated drives due to simulator sickness. The final sample included 17 participants (8 female).

Simulated drives and descriptions. The drives took place in a rural setting, with hills of varying elevations, curves, and straight segments incorporated in residential and wooded environments. A practice drive was designed to help the participant become comfortable with the simulations. Each of the experimental drives included a specific destination, directed by directional signs posted in the driving environment. Participants were to complete a baseline drive (no feedback), two feedback drives, and then a repeat baseline drive (no feedback). A simulated in-vehicle monitoring system was incorporated in the two feedback drives and an experimenter monitored the speed and triggered auditory messages when speeding thresholds were reached. Auditory messages were delivered from a smartphone, which was connected to a Bluetooth speaker, located on the right side of the simulator's dashboard.

Auditory messages. The auditory messages were designed to mimic some of the features in existing smartphone-based applications and to reflect young drivers' preferences collected in our interviews. They were categorized into two categories: positive and negative contents. Table 2 lists the messages, their associated tones, and conditions for delivering each message.

Table 2. Frequency and condition for which feedback messages were delivered

\begin{tabular}{|c|c|c|}
\hline Message & Condition When Delivered & $\begin{array}{c}\text { Limit to 'n' } \\
\text { times }\end{array}$ \\
\hline \multicolumn{3}{|c|}{ Positive Content-when safe speeds are shown } \\
\hline "Good Speed" -- neutral tone & $0-5 \mathrm{mph}<$ posted speed limit & 3 each drive \\
\hline "Great Driving" -- medium tone & 0-5 mph < posted speed limit; deliver after three "Good Speed" messages & 2 each drive \\
\hline $\begin{array}{l}\text { "You're an Excellent Driver" -- } \\
\text { emphasized tone }\end{array}$ & $\begin{array}{l}0-5 \mathrm{mph}<\text { posted speed limit for most of the drive; deliver only once at the } \\
\text { end of the second feedback drive }\end{array}$ & $\begin{array}{l}1 \text { at end of } 2^{\text {nd }} \\
\text { feedback drive }\end{array}$ \\
\hline \multicolumn{3}{|c|}{ Negative Content-when unsafe speeds are shown } \\
\hline "Slow Down" -- neutral tone & $5 \mathrm{mph}>$ posted speed limit for 5 seconds; deliver after the $5^{\text {th }}$ second & 3 each drive \\
\hline "Too Fast"-- medium tone & $\begin{array}{l}\text { Deliver after three "Slow Down" messages OR } \\
\text { if speed is } 10 \mathrm{mph}>\text { posted speed limit; deliver after the } 5^{\text {th }} \text { second }\end{array}$ & 2 each drive \\
\hline $\begin{array}{l}\text { "You Must slow down" -- } \\
\text { emphasized tone }\end{array}$ & $\begin{array}{l}\text { Deliver after two "Too Fast" messages OR } \\
\text { if speed is } 15 \mathrm{mph}>\text { posted speed limit; deliver after the } 5^{\text {th }} \text { second }\end{array}$ & 1 each drive \\
\hline "Bad Driving" -- stern tone & $\begin{array}{l}\text { Can be triggered anytime if unsafe/illegal conditions are observed, such as } \\
\text { running a stop sign and colliding with car or object/tree/pole/sign }\end{array}$ & Unlimited \\
\hline
\end{tabular}

Apparatus. The equipment used for this study included a high fidelity, fixed-base simulator (Realtime Technologies Inc. ${ }^{\circledR}$ ) and a mobile eye tracker (Applied Science Laboratories ${ }^{\circledR}$ Mobile Eye-XG). Data collected from the eye tracker are not reported here.

Procedures. After completing consent forms and eligibility checking, each participant was invited to sit in the simulator and be acquainted with the vehicle controls. An experimenter 
calibrated the eye tracker for each participant. Participants were instructed to follow driving directions and drive safely as if they were driving in the real world. Once the participant was comfortable in the car, the practice drive began. The baseline drive, two feedback drives, and repeated baseline drive followed the practice drive. The order of the two feedback drives was randomized. The participants were offered breaks between the drives. At the culmination of the drives, the experimenter debriefed the participants and distributed the compensation.

Data processing. Data collected from the driving simulator were merged with time stamps from the delivery of feedback messages. We focused on driver's velocity, braking, and acceleration patterns during 2-second and 5-second intervals before and after each feedback message.

\section{RESULTS- SIMULATOR STUDY}

Distributions of the direction and magnitude of changes in velocity, braking, and throttle between the averages during 2 -second and 5 -second before and after the delivery of feedback messages were examined. Table 3 lists the total of feedback messages (both positive and negative) as well as the breakdown of the specific contents and the resulting direction and magnitude of velocity change in the 2-second intervals before and after each message. We observed that the majority of messages triggered were positive ones; however, the resulting direction of velocity change was almost evenly distributed between reducing and increasing speeds. Therefore, the occurrences of receiving "Good speed" and "Great driving" (bolded in Table 3 ) were further analyzed by assessing the interactions between the positive content ( 2 levels) and feedback drive (2 levels).

Table 3. Occurrence of feedback messages and speed changes 2-seconds after receiving messages

\begin{tabular}{|c|c|c|c|c|c|c|}
\hline \multirow{2}{*}{$\begin{array}{l}\text { Message content } \\
\text { Positive message } \\
\end{array}$} & \multirow{2}{*}{$\begin{array}{l}\begin{array}{c}\text { Messages } \\
\text { delivered }\end{array} \\
\text { Total } 142 \\
\end{array}$} & \multicolumn{3}{|c|}{$\begin{array}{l}\text { Driver's direction of velocity change } \\
\text { (occurrence): }\end{array}$} & \multicolumn{2}{|c|}{$\begin{array}{l}\text { Driver's averaged magnitude } \\
\text { of velocity change }(\mathrm{m} / \mathrm{s}) \text { : }\end{array}$} \\
\hline & & reduced & increased & same & reduced & Increased \\
\hline "Good speed" & 101 & 58 & 41 & 2 & -1.39 & 1.62 \\
\hline "Great driving" & 39 & 20 & 19 & 0 & -2.27 & 1.62 \\
\hline "You are an excellent driver" & 2 & 2 & 0 & 0 & -0.6 & -- \\
\hline Negative messages & Total 21 & reduced & increased & same & reduced & Increased \\
\hline "Slow down" & 15 & 14 & 1 & 0 & -2.91 & 1.1 \\
\hline "Too fast" & 4 & 4 & 0 & 0 & -2.47 & -- \\
\hline "You must slow down" & 2 & 2 & 0 & 0 & -3.67 & -- \\
\hline
\end{tabular}

For both 2- and 5-second intervals, the distributions of changes in velocity were slightly negatively skewed (-1.38 and -1.08 , respectively) and the changes in throttle were slightly positively skewed (1.08 and 1.12, respectively). A few transformations were tried but none of them improved the skewness, therefore, the original data were used for further analysis. For both 2- and 5-second intervals, the distributions of changes in braking were positively skewed (3.21 and 4.14 , respectively) and a natural logarithm transformation was used to improve the normality of the distribution (skewness $=-.87$ and -.68). Repeated measures ANOVAs showed that there were no statistically significant differences in the magnitude of change in velocity, braking, and throttle, between the two contents of positive message and between the two feedback drives. These results suggest that the magnitude of change in vehicle control was similar across the two feedback drives and upon receiving the two positive contents. 
Since every participant received three "Good speed" messages in each of the experimental drives, we tested the effect of the three occurrences on velocity, braking, and throttle change across the two feedback drives and did not observe any significant effects, suggesting that participants did not react differently across the three "Good speed" messages.

\section{DISCUSSION}

The study was designed to better understand young drivers' perspectives on in-vehicle monitoring technologies. Previous studies have shown mixed perceptions about such systems from teens and parents, although there was evidence of effectiveness for behavioral changes. We argued that it is important to associate learning with positive outcomes by using reinforcement techniques such as offering positive recognition, instead of only focusing on alert-based and punishment-oriented learning experiences. One of the reasons for the favorable effect of positive reinforcement on young drivers is that recognizing their behaviors positively will strength the association between intentions and learning outcomes (Bandura, 1969; Skinner, 1953).

Our interview results suggested that a point system where young drivers could earn or lose points is favorable. They also recognized areas for improvement (i.e., following speed limit, appropriate braking, and safe headway distance) for both of the feedback contents (positive and negative). Their preferences for the delivery timing of feedback suggest that real-time recognition/warning delivered auditorily would be most helpful.

A subsequent feasibility driving simulator study was designed to incorporate key findings from the interviews (i.e., real-time auditory feedback on speeding) and examine the effect of such reinforcement techniques on speed management behaviors. The preliminary results showed almost even distribution of the direction of velocity change upon receiving positive feedback messages. Our analyses were guided by the occurrences of feedback messages and the lack of statistical significance needs to be explained carefully. We suspected that environmental conditions may be a factor, and participants might responded differently when the message was delivered during an uphill, downhill, curve, or straight road. We will examine the patterns of change before and after each message by terrains and speed limit zones. In addition, we plan to replicate the simulator study and fine-tune the feedback mechanisms with a larger sample size.

Limitations. The simulator study was exploratory in nature and was purposely design to assess the feasibility of incorporating interviewees' preferences on feedback messages while taking into account the kind of short messages being used in existing smartphone-based applications in the market. We did not include peer passengers or other kind of reinforcement techniques, nor did we compare between age groups or between intervention and control groups. Future research should compare the presence of feedback messages in conjunction with incentive-based positive reinforcement techniques (Volpp et al., 2009) as parts of speeding mitigation strategies. Parents, in-vehicle monitoring devices such as smartphone-based applications, and a third party that can provide financial incentives such as insurance companies should work together to define a timeline and goals for continued learning and education beyond licensure to ensure the young novice drivers can work on improving their driving skills in a positive and welcoming environment. 


\section{ACKNOWLEDGEMENTS}

The authors would like to acknowledge the National Science Foundation (NSF) Center for Child Injury Prevention Studies at the Children's Hospital of Philadelphia (CHOP) for sponsoring this study and its Industry Advisory Board (IAB) members for their support, valuable input and advice. The views presented are those of the authors and not necessarily the views of CHOP, the NSF, or the IAB members.

\section{REFERENCES}

Bandura, A. (1969). Principles of behavior modification. New York: Holt, Rinehart and Winston, Inc.

Cestac, J., Paran, F., \& Delhomme, P. (2011). Young drivers' sensation seeking, subjective norms, and perceived behavioral control and their roles in predicting speeding intention: How risk-taking motivations evolve with gender and driving experience. Safety Science, 49(3), 424-432.

Farmer, C. M., Kirley, B. B., \& McCartt, A. T. (2010). Effects of in-vehicle monitoring on the driving behavior of teenagers. Journal of Safety Research, 41(1), 39-45.

Foss, R. D., Martell, C. A., Goodwin, A. H., \& O'Brien, N. P. (2011). Measuring changes in teenage driver crash characteristics during the early months of driving. Washington, DC: AAA Foundation for Traffic Safety.

Gesser-Edelsburg, A., \& Guttman, N. (2013). "Virtual" versus "actual" parental accompaniment of teen drivers: A qualitative study of teens' views of in-vehicle driver monitoring technologies. Transportation Research Part F-Traffic Psychology and Behaviour, 17, 114124.

Horvath, C., Lewis, I., \& Watson, B. (2012). Peer passenger identity and passenger pressure on young drivers' speeding intentions. Transportation Research Part F-Traffic Psychology and Behaviour, 15(1), 52-64.

McCartt, A. T., Farmer, C. M., \& Jenness, J. W. (2010). Perceptions and experiences of participants in a study of in-vehicle monitoring of teenage drivers. Traffic Injury Prevention, 11(4), 361-370.

McGehee, D. V., Raby, M., Carney, C., Lee, J. D., \& Reyes, M. L. (2007). Extending parental mentoring using an event-triggered video intervention in rural teen drivers. Journal of Safety Research, 38(2), 215-227.

Simons-Morton, B. G., Bingham, C. R., Ouimet, M. C., Pradhan, A. K., Chen, R., Barretto, A., \& Shope, J. T. (2013). The effect on teenage risky driving of feedback from a safety monitoring system: A randomized controlled trial. Journal of Adolescent Health, 53(1), 21 26.

Skinner, B. F. (1953). Science and human behavior. New York: The Macmillan Company.

Toledo, T., Lotan, T., Taubman-Ben-Ari, O., \& Grimberg, E. (2012). Evaluation of a program to enhance young drivers' safety in Israel. Accident Analysis and Prevention, 45, 705-710.

Volpp, K. G., Troxel, A. B., Pauly, M. V., Glick, H. A., Puig, A., Asch, D. A., . . AudrainMcGovern, J. (2009). A randomized, controlled trial of financial incentives for smoking cessation. New England Journal of Medicine, 360(7), 699-709. 